\title{
Throughput Port Demand Forecasting
}

\author{
Jeffrey E Jarrett* \\ Professor of Management Science and Finance, University of Rhode Island, Kingston- 02852, USA
}

\begin{abstract}
Forecasts are numerical estimates of the future levels of sales, demand, inventories, costs imports, exports and prices, among others. For a firm, industry, a sector of the economy or the aggregate economy let alone factors such as international trade and finance. The objective is to assist management in planning, budgeting, marketing efforts, materials requirements, sustainability, the refuse from power production and efforts to restrain the effects of pollution on climate change.

We indicate several procedures which are relatively easier to compute, more accurate and not dependent on finding predictor (or explanatory) variables which have coefficients of correlation which are small. By using one set of data, (port demand in units), we do short-term forecasting of up to twelve periods which are less data dependent and produce forecasts which do not require a huge investment in computer and personnel time.
\end{abstract}

Keywords: Time series; Autocorrelation function (ACF); Partial autocorrelation function (PACF); Forecast modeling; Ljung-box statistic; Decomposition

\section{Introduction}

Traditional methods to forecast port traffic volumes include basing projections based on GDP over time or desk and field research often how it relates to changes in the cargo port and domestic and international shipping prices. Although each method has its advantages and disadvantages, our purpose here is to determine if parsimonious forecasting models can prove to be superior to the common methods. Both of the traditional procedures laid themselves open to major error.

Importance of port traffic forecasting associated or not by political, economic

One of the best ways for successful management of certain transport companies is traffic demand planning. Demand is one of the most important aspects of business economics. Mismatch between supply and demand leads to a number of problems. Therefore, in case of port operations, higher supply than demand leads to the failure in the utilization of port infrastructure and superstructure, and to the lack of cost-effectiveness. When the demand for port services exceeds the supply, there comes to congestion of port facilities, an increase in costs of ships and losses of time due to waiting. Since the traffic capacity is not flexible and its construction and development require substantial financial resources in order to be payable, supply must be designed in accordance with the anticipated demand in the future. Therefore, to avoid the consequences of non-compliance of port supply and demand, and to create a basis for sizing supply, there is a need for demand forecasting of port services.

For future demand forecasting, the primary precondition is the construction and/or modernization of the future port infrastructure and superstructure. Since the port service cannot be stored, the port offer needs to be in line with the current demand. The analysis of relevant materials regarding traffic forecasting of demand for port services, particularly the demand for port services can change rapidly with the change relating to priorities set by governments, government agencies, demand conditions set in motion by buyers and sellers of the materials being transported by the local supply chain.

\section{Previous study}

Cargo port activity is a time series with random fluctuations associated with political, economic and natural forces affecting small deviations. Typically (noted above), one forecasts using linear or nonlinear methods associated with an important economic indicator (GDP). Typically, one focuses on port traffic demand in three areas $[1,2]$.

First, making local improvements on the existing prediction methods, so we can obtain the prediction result which is closer to the true value. By adjusting the inflection point of the Logistic curve, researching the methods of blur compensation and estimation of optimism and pessimism on production trends including Logistic curve prediction methods.

Second, some experts predict the cargo port handling activity by referring to methods from other applications including the use of econometrics, control theory and related methods. Others have produced dynamic PHQDF models to predict the cargo port activity, which systematically combines the historical trend of cargo activity, the socio-economic development of the hinterland, politics, policy, and psychological and technical factors. The example tests show that the model has higher prediction precision and better prediction results than the models suggested above.

Third, have the raw data processed, and through selecting appropriate explanatory variables from a plurality of explanatory variables, we may obtain better forecast results. Regression models to predict the cargo port activity are not uncommon. As results often indicate, when one utilizes mathematical models to predict, the most critical aspect is that the accuracy of the model, relationship of the response variable and predictor's variables and the quality of data is most important. The complexity of the model, especially in the case of only having small amounts of historical data is of lesser importance. Some examples of forecasting port activity by many researchers [3-9]. In each application, they use very large amounts of data to predict port capacity, tonnage and other measures of activity including correlated variables.

*Corresponding author: Jeffrey E. Jarrett, Professor of Management Science and Finance, University of Rhode Island, Kingston-- 02852, USA, Tel: +1 401-874-1000; E-mail: jeffreyejarrett@gmail.com

Received September 03, 2015; Accepted October 12, 2015; Published October 16, 2015

Citation: Jarrett JE (2015) Throughput Port Demand Forecasting. Int J Econ Manag Sci 4: 293. doi:10.4172/21626359.1000293

Copyright: ( 2015 Jarrett JE. This is an open-access article distributed under the terms of the Creative Commons Attribution License, which permits unrestricted use, distribution, and reproduction in any medium, provided the original author and source are credited. 


\section{Methodology}

\section{An example using a parsimonious time series method}

First, we examine the time series of a sample of data for a Chinese port to observe the dimensions of a time series plot of data. Figure 1 contains the plot of six years by month of port traffic at a river port in the People's Republic of China (PRC). The time series plot shows the ups and downs of the data indicating a long term trend in the time series with variation from month to month. Since our purpose is to simplify the forecasting to the use of a univariate autoregressivemoving-average (ARIMA) model we will further examine the data if it is necessary to do additional predictions Figure 1.

By additional steps, we will see if we can model port traffic efficiently without great time and cost associated with sophisticated models. The additional steps will identify the specification of the ARIMA model. Step 1 is to determine the ARIMA function by computing the autocorrelation function (ACF) and partial autocorrelation function (PACF). These functions are drawn in Figures 2 and 3. Figure 2 contains the autocorrelation function for the time series data for the port traffic of Figure 1. Note, the function indicates a slow decline to about lag 6 indicating that the model is AR. To determine the appropriate number of AR parameters to estimate, we draw and obtain the partial autocorrelation function (PACF) of the same data in Figure 3. The partial autocorrelation function contains a large value at lag 1 (approximately, 0.8) which is well above the 95\% significant limit in Figure 3. Hence, the number of AR parameters to be estimated is 1 .

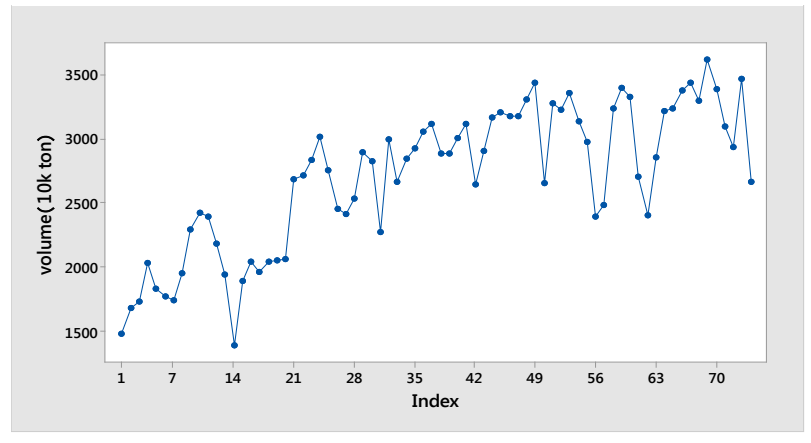

Figure 1: Time series plot of port traffic.

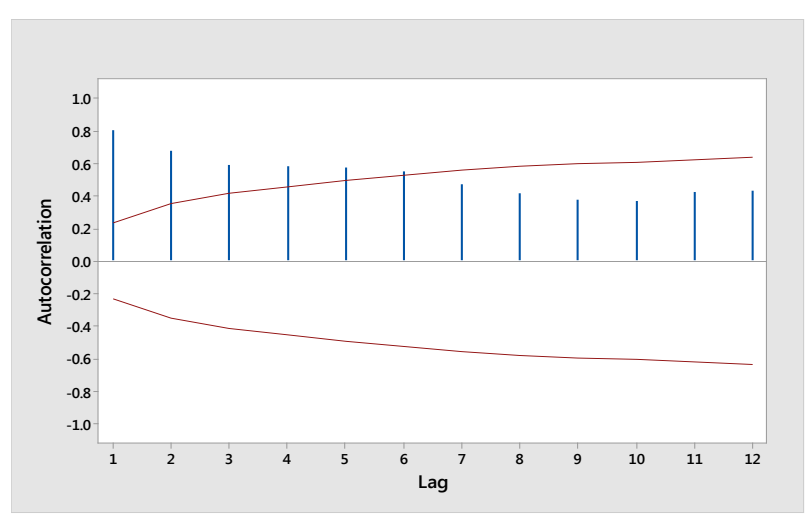

Figure 2: ACF of volume.

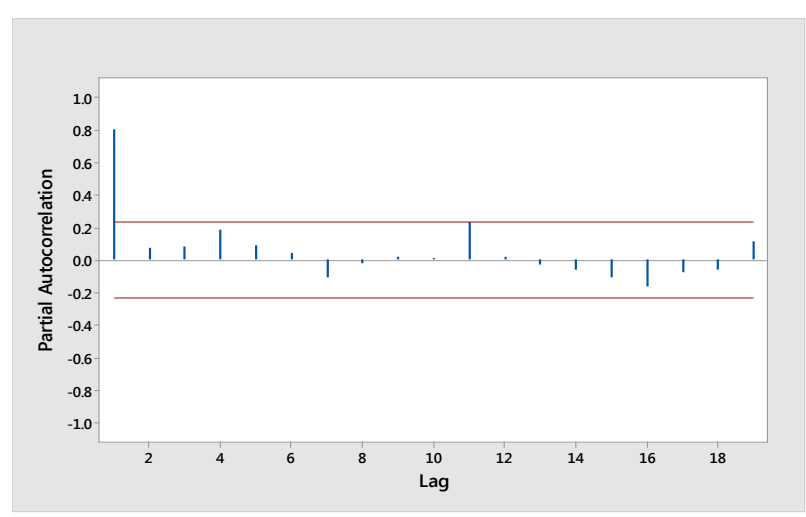

Figure 3: PACF of volume.

\begin{tabular}{|c|c|c|c|c|}
\hline Type & Coefficient & St. Error & T & P \\
\hline AR (1) & 0.8624 & 0.0624 & 13.82 & 0.000 \\
\hline Constant & 359.82 & 35.82 & 10.03 & 0.000 \\
\hline Mean & 2610.6 & 260.2 & & \\
\hline Modified Box-Pierce (Ljung-Box) Chi-Square statistic \\
\hline Lag & 12 & 24 & 36 & 48 \\
\hline Chi-Square & 12.9 & 21.5 & 32.7 & 46.2 \\
\hline DF & 10 & 22 & 34 & 46 \\
\hline P-Value & 0.228 & 0.492 & 0.532 & 0.465 \\
\hline
\end{tabular}

Number of observations: 74

Residuals: $\quad$ SS $=6711497$ (back forecasts excluded)

$\mathrm{MS}=93215 \mathrm{DF}=72$

Table 1: Final Estimates of Parameters from Minitab17.

\section{Results}

\section{The ARIMA model and analysis}

The ARIMA modeling in the first two steps are discussed in a wide amount of literature including the original developers Box and Jenkins [10] and explained also in many practitioner oriented literature include Makridakis et al. and Jarrett [11]. Using Minitab (vers 17) we find the following estimates of the final parameter of the AR (1) model.

In Table 1 of results, we obtain the model having 359.31 as the estimate of the constant and 0.8264 as the estimate of the first lag. In both the cases of the estimate of the constant and first AR coefficient, we observe $p$-values of 0.000 for the hypotheses that the parameters for the constant and AR (1) are equal to zero. For the modified Ljung-Box statistic indicates that the parameter test tends also to indicate there is no serial correlation in the autocorrelation of the residuals.

To finish the validation of the modeling process we calculate the autocorrelation of the residuals from forecast. Again the p-values indicate that the likelihood that we cannot reject the hypothesis that the model is adequate. What do we mean by adequate?

The above plot of time series for volume ( $10 \mathrm{k}$ ton) indicate the direction of port demand through time period 86 (12 periods ahead. Also, 95\% confidence interval estimates ahead to period 86 are also shown. Planners and managers with 95 percent certainty are able to know the limits of the prediction. In Figure 4, one observes the value of the mean square error $(\mathrm{MS}=93215)$ and degrees of freedom associated with the predictive model. If we find a model that contains a smaller MS, that model could predict with uncertainty a better result. For 


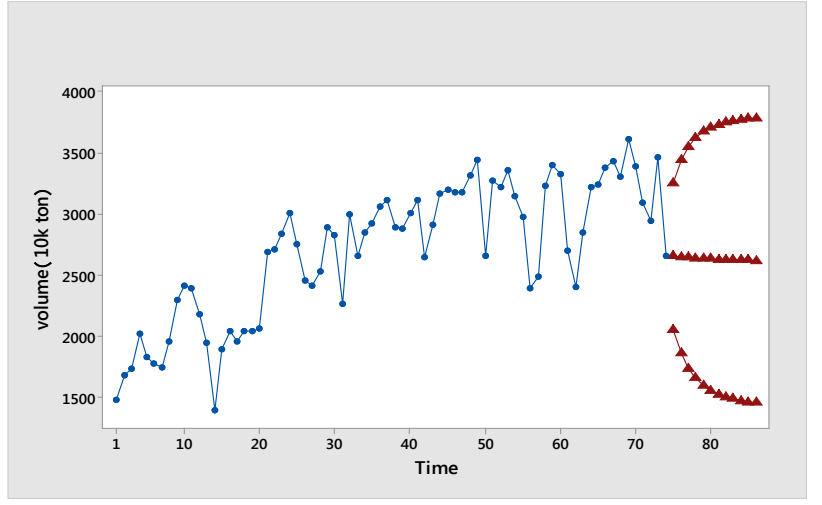

Figure 4: Time series plot for volume.

\begin{tabular}{|l|c|c|c|c|}
\hline \multicolumn{1}{|c|}{ Type } & Coef & SE Coef & T & P \\
\hline AR (1) & 0.7776 & 0.1229 & 6.33 & 0.000 \\
\hline AR (2) & 0.1055 & 0.1222 & 0.86 & 0.391 \\
\hline Constant & 303.40 & 36.11 & 8.40 & 0.000 \\
\hline Mean & 2594.3 & 308.8 & & \\
\hline Modified Box-Pierce (Ljung-Box) & Chi-Square statistic & \\
\hline Lag & 12 & 24 & 36 & 48 \\
\hline Chi-Square & 15.5 & 23.4 & 34.5 & 50.4 \\
\hline DF & 9 & 21 & 33 & 45 \\
\hline
\end{tabular}

Number of observations: 74

Residuals: $\mathrm{SS}=6663734$ (back forecasts excluded)

$\mathrm{MS}=93855 \mathrm{DF}=71$

Table 2: Result of AR (2) Model Estimation and Final Estimates of Parameters.

example, we may include a second autoregressive term in the forecast mode denoted AR (2). The result would be in Table 2 below.

Note, in Table 2, the AR (2) parameter estimates has p-value of 0.391 indication that one would not reject the hypothesis that the coefficient for this term is 0 . By so doing, we would eliminate this value as an explanatory variable. Hence, we ar reduced to the AR (1) term. Also, the MS of the new model is 93855 which is larger than the MS of the AR (1) model which is 93218 [10-12]. The results noted in Table 2 if further examined by the autocorrelation function of the residuals would also indicate that the above estimated model would indicate that the model would not pass the test that autocorrelation is not present in the error terms. Hence, one would be better off by using the AR (1) model identified in the first step of the modeling process.

\section{Forecasting with time series decomposition}

Another method of forecasting which does not require finding highly correlated predictor variables is referred to as time series decomposition. Decomposition separates the time series from linear trend and seasonal components, as well as error, and to produce forecasts. For example, you might want to predict the port demand over the next twelve months data collected over the past 72 months.

You can use decomposition when you have data with either no trend or constant trend; have data with constant seasonal pattern; the seasonal component is either additive or multiplicative with the trend; and you want to make intermediate or long range forecasting. The methodology of time series decomposition developed around 1900 is one of the oldest methods for forecasting and is fully described in
Jarrett [11] chapter 3 and many other books on predictive methods. Using conventional software on PC's and desk computers (Minitab), one may illustrate and compute the forecast. The result for our six years of monthly data for port traffic is illustrated in Figure 5. The figure contains the original 72 monthly data on port traffic and the 12 month period ahead forecasts. The plot contains actuals, fits, trend and forecasts and is denoted on the plot and variable definition box. Also, noting the accuracy measures is MSD (mean square deviation which MS in the previous figures) which is 92118.9. This value is smaller than the MS value. Hence the decomposition analysis is more accurate than either of the autoregressive models developed for these data previous.

\section{Conclusions and Future Research}

The analysis of the data indicates that ARIMA modeling and Time Series Decomposition produce useful results. Note in examining the final estimates of the AR (2) model (Table 2) the AR (2) parameter yielded a t-value of 0.86 corresponding to a $p$-value of 0.391 . Hence, a second round of ARIMA modeling without the AR (2) parameter estimate would yield better results with only the constant and AR (1) parameter included in the forecasting model. Not reported in our tables but calculated the final MS estimate was smaller than for the AR (2) model. The extant of the decrease was not huge but the conclusion was that better improvement in the model forecasting was possible.

By examining Figure 5, the Time Series decomposition method also produced very good results which would fit a use trend through the data points and when reintroducing seasonality into the final forecast would result in a set of forecasts that closely model the pattern existing in the historical time series. The great ad vantages of these methodologies is that the methods do not require searching for external environmental variables which relate to throughput demand making the process of prediction and planning more difficult. Simple methods as noted in the analysis are available on common computer software. The methods do not require huge data banks or retrieval of data which may be from suspect sources. Jarrett, Zhang and Pan [1113] noted that the use of freight rates in predicting cargo port volume but do require a very sophisticated model using a two parameter exponentially weighted moving-average model requiring much more analysis and data requirement than utilized in this study.

Our results do not stop one from seeking alternative, Jiawei, Lizhi, Xun and Shouyang $[14,15]$ proposed in utilizing CDE-MPR methodology. Their results apply to multi-port regions in South China (PRC). They demonstrated that a feasible method to forecast and analyze maritime logistics in a multi-port regions. The methods

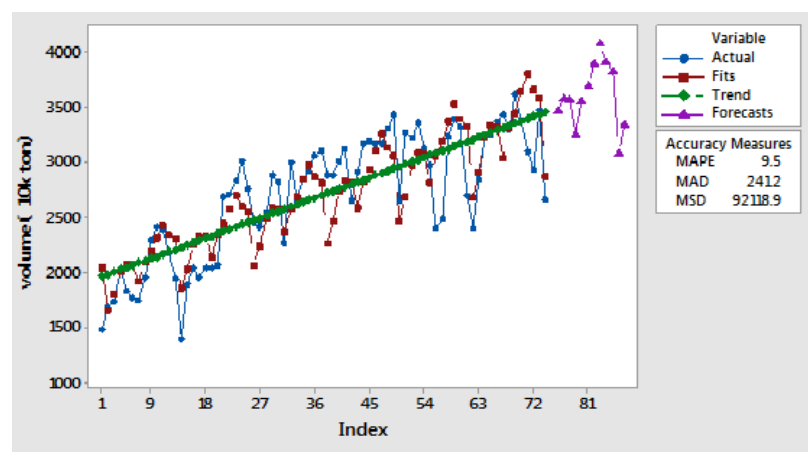

Figure 5: Time series decomposition plot for volume (10k ton) multiplicative model. 
consist of many choices since there are many models to be included in the process. Their approach is promising but restricted to multi-port regions. The methods also are encouraging in designing and planning supply-chains.

\section{References}

1. Bakshi G, Panayotov G, Skoulakis G (2010) The Baltic Dry Index as a Predictor of Global Stock Returns, Commodity Returns, and Global Economic Activity.

2. Sun $L$ (2010) The research on a double forecasting model of port cargo throughput. World Journal of Modeling and Simulation 6: 57-62.

3. Sun L, Wang Z (2007) Studying the Probability Distribution of the port handling capacity in the port. Proceedings of SSMSSD 6: 1869-1873.

4. Yuan E, Xie Y (2004) On Ports Throughput Probability Forecasting Model. Port and Waterway Engineering 33: 1028-1031.

5. Cheng L, Wong Y (1997) Port Facilities and Container Handling Services: The Hong Kong Economic Policy Studies, City University of Hong Kong Press, Hong Kong.

6. Sun Y, Zheng G (2007) Research on Forecast of Port Throughput Capacity Based on Grey Theory. China Water Transport 5: 160-162.
7. Yang S, Guan K (2005) Application of Spreadsheet to Forecasting Port Throughput. Wuhan Journal of University of Technology.

8. Hui E, Seabrooke W, Woong K (2004) Forecasting Cargo Throughput for the Port of Hong Kong: Error Correction Model Approach. Journal of Urban Planning and Development 130: 195-203.

9. Lam WHK, Pan LP Ng; Seabrooke W, Hui ECM (2004) Forecasts and Reliability Analysis of Port Cargo Throughput in Hong Kong. Journal of Urban Planning and Development 130: I33-144.

10. Box G, Jenkins G $(1970,1976)$ Time Series Analysis: Forecasting and Control. Operational Research Quarterly 22:199-201.

11. Jarrett J (1991) Business Forecasting Methods, (2ndedn), Basil Blackwell: Oxford.

12. Pankratz A (1983) Forecasting with Univariate Box-Jenkins Models: Concepts and Cases, John Wiley and Sons, New York.

13. Xu C, Yan Y, Zhang P (2006) Prediction Model of Port Throughput Based on System Dynamics. Port and Waterway Engineering 32: 26-29.

14. Chen N, Zhu M, Yu Z (2005) Comparing Two Forecast Models of Ports Handling Capacity. Journal of Wuhan University of Technology 27: 77-79.

15. Wang C (2006) Port Handling Capacity Forecasting Based on Unascertained Number Regressive Model Communications Standardization. 